\title{
Número e espaçamento entre hastes de guia de onda para medida da umidade do solo com TDR
}

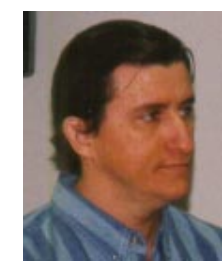

\author{
Eugênio F. Coelho ${ }^{1}$, Ranulfo C. Caldas ${ }^{1}$, Delfran B. dos Santos ${ }^{2}$ \& Carlos A. da S. Ledo ${ }^{1}$ \\ 1 EMBRAPA Mandioca e Fruticultura. CEP 44380-000, Cruz das Almas, BA. E-mail: ecoelho@cnpmf.embrapa.br (Foto) \\ 2 Doutorando, DEA/UFV. CEP 36570-000, Viçosa, MG. E-mail: delfran@zipmail.com.br \\ Protocolo 167 - 11/11/2002 - Aprovado em17/7/2003
}

\begin{abstract}
Resumo: Com este trabalho, objetivou-se avaliar o desempenho de diferentes modelos matemáticos para ajuste dos dados de umidade, em função da constante dielétrica aparente do solo, e a viabilidade de uso de guias de onda de duas e três hastes, com diferentes espaçamentos. Amostras de solo deformadas foram acondicionadas em segmentos de tubos de PVC de 0,075 m de diâmetro. Construíram-se 24 guias de onda com capacitor e 24 guias de onda sem capacitor, sendo que, para cada tipo, 12 guias de onda de duas hastes e 12 de três hastes, com espaçamento entre hastes de 0,009 a 0,022 m, de impedâncias previamente determinadas, foram inseridas no solo, após a saturação em cada segmento de tubo. Dados de umidade do solo determinados por gravimetria e da constante dielétrica aparente obtidos pelo analisador Trase System, foram tomados em cada recipiente durante a secagem do solo de 0,31 a $0,13 \mathrm{~m}^{3} \mathrm{~m}^{-3}$. Cinco modelos matemáticos foram ajustados aos dados de umidade em função da correspondente constante dielétrica aparente do solo e o efeito dos modelos na obtenção da umidade do solo, em função da constante dielétrica aparente, foi avaliado estatisticamente. O modelo de Malicki foi o de melhor ajuste dos dados da constante dielétrica aparente, em função da umidade do solo. As guias de onda de três hastes, de diâmetro 0,003 m, comprimento 0,15 m, com espaçamentos entre hastes de 0,017 m, foram as de melhor desempenho, enquanto as guias de onda de três hastes sem capacitor indicaram melhor desempenho na determinação da umidade do solo, comparadas com as de duas hastes sem capacitor. As guias de onda de três hastes sem capacitor apresentaram melhor desempenho na determinação da umidade do solo que as de três hastes com capacitor.
\end{abstract}

Palavras-chave: calibração, guia de onda, umidade, reflectometria no domínio do tempo

\section{Number and spacing between wave guide rods for measurement of soil water content with TDR}

\begin{abstract}
The objective of this study was to evaluate the performance of different mathematical models to adjust the humidity data as a function of the apparent dielectric constant of the soil and to evaluate the feasibility of the use of wave guides of two and three rods with different spacings. Disturbed soil samples were packed in PVC tube segments of $0.075 \mathrm{~m}$ diameter. Two sets of 24 have guides were constructed. One of this sets had a capacitor. In each set one half of the wave guides had two rods and the other half contained three rods. The rod spacing varied from 0.009 to $0.022 \mathrm{~m}$. Soil water content data from gravimetry and soil bulk dielectric constant values from Trase System analyzer were collected during drying process with water content values ranging from 0.31 to $0.13 \mathrm{~m}^{3} \mathrm{~m}^{-3}$. Five mathematical models were fitted to water content and bulk dielectric constant data. The Malicki's model was the most adequate for estimating soil water content as a function of bulk dielectric constant. The wave guides with three rods $0.017 \mathrm{~m}$ apart from each other showed the best performance. The three-rod wave-guides without capacitor performed better for water content determination than the two-rod wave-guides without capacitor. The three-rod wave-guides without capacitor performed better than three-rod wave-guides with capacitor.
\end{abstract}

Key words: calibration, wave guide, soil water content, time-domain-reflectometry

\section{INTRODUÇÃO}

A umidade do solo constitui-se num dos parâmetros básicos mais necessários dentro do sistema solo-água-plantaatmosfera, na avaliação e no monitoramento dos sistemas hidrológicos. Sua determinação indireta, pela técnica da reflectometria no domínio do tempo - TDR, é precisa, não destrutiva, permite a determinação de umidade em tempo real e a continuidade e automação na coleta dos dados.

As guias de onda usadas para transmitir o pulso eletromagnético do analisador de umidade podem ser construídas de diferentes maneiras, dependendo do objetivo. Existem guias 
de onda contínuas e segmentadas, com diferentes comprimentos e número de hastes (Conciani et al., 1997). As contínuas integram a umidade ao longo das hastes, num valor médio da camada de solo e as segmentadas permitem medir-se umidade em diferentes camadas do solo (Souza et al., 2000). Quanto ao número de hastes, Zegelin et al. (1989) iniciaram o uso das guia de ondas multi-hastes, dentre as quais a de três hastes tem sido a mais utilizada. As guia de ondas de TDR de duas hastes devem conter casadores de impedância; entretanto, diversos autores (Malicki \& Skierucha, 1989; Malicki et al., 1992; Kelly et al., 1995; Patterson \& Smith, 1981) as têm usado sem esses dispositivos.

Os usuários da técnica da TDR têm usado guias de onda de construção própria, por terem custo mais baixo que as guias comercializadas. No caso da TDR Trase system $1^{*}$, conseguemse leituras diretas pelo aparelho com inserção de capacitores de 3,9 pF no início das hastes (Coelho et al., 2001; Souza et al., 2001).

Na construção das guias de onda o número, o espaçamento e o comprimento das hastes são os fatores variáveis. O desenho dessas guias de onda tem sido padronizado em alguns aspectos, mas não quanto ao espaçamento entre hastes. Knigh (1992) sugere que o espaçamento entre hastes de uma guia de onda deve estar relacionado ao diâmetro das mesmas, conforme a razão diâmetro/espaçamento > 0,1 para evitar concentração de energia em volta das hastes.

O conhecimento do número e espaçamento entre hastes de guias de onda com e sem capacitor, que permitam a determinação adequada da umidade do solo, para uso com o analisador Trase System 1, poderá facilitar a construção das guias de onda pelos usuários.

Este trabalho teve como objetivo avaliar o desempenho de diferentes modelos para determinação de umidade em função da constante dielétrica aparente do solo e também a viabilidade de uso de guias de onda com e sem capacitor, de duas e três hastes, sob diferentes espaçamentos.

\section{MATERIAL E MÉTODOS}

Amostras de solo deformadas foram retiradas em um Latossolo Amarelo Distrófico, com as seguintes características físicas: $590 \mathrm{~g} \mathrm{~kg}^{-1}$ de areia total, $95 \mathrm{~g} \mathrm{~kg}^{-1}$ de silte, $315 \mathrm{~g} \mathrm{~kg}^{-1} \mathrm{de}$ argila, densidade do solo de $1,33 \mathrm{~kg} \mathrm{dm}^{-3}$, densidade de partículas $2,50 \mathrm{~kg} \mathrm{dm}^{-3} \mathrm{e}$ porosidade total $32 \%$. As amostras deformadas foram destorroadas e secadas ao ar sendo, em seguida, acondicionadas em 48 segmentos de tubos de PVC, previamente pesados, de $0,25 \mathrm{~m}$ de comprimento e diâmetro de $0,075 \mathrm{~m}$ até a altura de $0,20 \mathrm{~m}$. Usou-se uma tela fina previamente pesada, que foi colada à parede da parte inferior do segmento de tubo para evitar perda de solo. O solo dentro dos segmentos de tubo foi saturado durante $48 \mathrm{~h}$.

Foram confeccionadas 48 guias de onda no Laboratório de Física de Solos da Embrapa Mandioca e Fruticultura, das quais 24 unidades tiveram um capacitor de $3,9 \mathrm{pF}$ (Coelho et al., 2001) no início das hastes de aço inox de $0,003 \mathrm{~m}$ de diâmetro. Determinou-se a impedância característica, $\mathrm{Z}_{0}$, de todas as guias de onda, conforme Zegelin et al. (1989):

$$
\mathrm{Z}_{0}=\mathrm{Z}_{\mathrm{u}} \sqrt{\varepsilon}\left(\frac{1+\rho_{\mathrm{r}}}{1-\rho_{\mathrm{r}}}\right)
$$

em que:

$$
\begin{array}{ll}
\mathrm{Z}_{\mathrm{u}} & \text { - Impedância do cabo co-axial, } \Omega \\
\varepsilon & \text { - constante dielétrica aparente do meio, no caso água } \\
& \text { destilada } \varepsilon=81,\left(25^{\circ} \mathrm{C}\right) \\
\rho_{\mathrm{r}} & \text { - coeficiente de reflexão, determinado em função da } \\
& \text { amplitude do sinal gerado pela TDR }\left(\mathrm{V}_{0}\right) \text { e da ampli- } \\
& \text { tude do sinal mínima detectada antes da reflexão do } \\
& \text { pulso eletromagnético }\left(\mathrm{V}_{1}\right), \rho_{\mathrm{r}}=\left(\mathrm{V}_{1} / \mathrm{V}_{0}\right)-1
\end{array}
$$

De cada 24 unidades, com ou sem capacitor, foram construídas 12 unidades com duas hastes e 12 unidades com três hastes. Para cada tipo de guia de onda foram testados quatro espaçamentos entre hastes: $0,009,0,013,0,017 \mathrm{e} 0,022 \mathrm{~m}$.

$\mathrm{O}$ experimento seguiu um delineamento experimental inteiramente casualizado em esquema fatorial $2 \times 2 \times 4$, com três repetições, sendo os fatores o tipo de guia de onda (com e sem capacitor), o número de hastes (duas e três hastes) e o espaçamento entre hastes.

As guias de onda foram pesadas e inseridas no solo úmido durante a saturação. Após a instalação das antenas, os recipientes foram mantidos com a superfície do solo exposta, para permitir perda de água apenas por evaporação. Os segmentos de tubo contendo solo e guias de onda foram pesados diariamente, desde a saturação $\left(0,31 \mathrm{~m}^{3} \mathrm{~m}^{-3}\right)$ até a umidade $0,13 \mathrm{~m}^{3} \mathrm{~m}^{-3}, 62$ dias após. Durante cada pesagem, foi feita a leitura na TDR Trase 1, obtendo-se o tempo de propagação do pulso eletromagnético e, conseqüentemente, a constante dielétrica aparente do solo, $\varepsilon_{\mathrm{b}}$, da qual indiretamente se estimou a umidade do solo.

No final de 62 dias após a saturação, retirou-se a guia de onda e a tela da coluna de PVC contendo o solo, sendo esta levada à estufa, a temperatura de $105{ }^{\circ} \mathrm{C}$, durante o tempo necessário para se atingir peso constante. Com base no peso seco da amostra somado aos pesos do segmento de tubo, da tela e da guia de onda, e pelas pesagens diárias determinaramse os teores de água correspondentes a cada pesagem (Coelho et al., 2001).

Os dados da constante dielétrica aparente, correspondente para cada umidade da amostra, foram calculados conforme Or $\&$ Wraith (1994) e, juntamente com os da umidade gravimétrica, foram usados para calibração das guias de onda de TDR, por meio de modelos físicos e empíricos.

Avaliaram-se os modelos de Roth et al. (1990), Malicki et al. (1996), Whalley (1993), Topp et al. (1980) e outros modelos foram também ajustados aos dados pelo aplicativo "Table curve 2D", tendo-se como resultado num modelo potencial, também usado para obtenção da umidade, em função da constante dielétrica aparente.

O modelo de Roth et al. (1990) considera a constante dielétrica aparente como uma resultante de componentes da matriz do solo $\left(\varepsilon_{\mathrm{s}}\right)$, do ar $\left(\varepsilon_{\mathrm{a}}\right)$ e da água $\left(\varepsilon_{\mathrm{w}}\right)$ :

$$
\varepsilon^{\alpha}=\theta \varepsilon_{\mathrm{w}}^{\alpha}+(1-\phi) \varepsilon_{\mathrm{s}}^{\alpha}+(\phi-\theta) \varepsilon_{\mathrm{a}}^{\alpha}
$$


em que:

$\phi \quad$ - corresponde à porosidade total, $\mathrm{m}^{3} \mathrm{~m}^{-3}$

$\theta \quad$ - à umidade do solo, $\mathrm{m}^{3} \mathrm{~m}^{-3}$

$\alpha \quad$ - leva em conta os efeitos do arranjo geométrico dos componentes do meio na constante dielétrica aparente aparente

Malicki et al. (1996) propôs um modelo relacionando a umidade e a constante dielétrica aparente com inclusão da densidade do solo, do tipo:

$$
\theta=\frac{\varepsilon^{0,5}-0,819-0,168 \rho_{\mathrm{b}}-0,159 \rho_{\mathrm{b}}{ }^{2}}{7,17+1,18 \rho_{\mathrm{b}}}
$$

em que:

$$
\rho_{\mathrm{b}} \quad \text { - densidade do solo, } \mathrm{Mg} \mathrm{m}^{-3}
$$

O modelo de Topp et al. (1980) consiste numa polinomial do terceiro grau para estimativa dos teores de água, como função da constante dielétrica aparente:

$$
\theta_{\mathrm{v}}=-5,3 \times 10^{-2}+2,92 \times 10^{-2} \varepsilon-5,5 \times 10^{-4} \varepsilon+4,3 \times 10^{-6} \varepsilon^{3}
$$

Whalley (1993) propôs um modelo do tipo:

$$
\sqrt{\varepsilon}=\theta\left(\sqrt{\varepsilon_{\mathrm{w}}}-1\right)+\frac{\rho_{\mathrm{b}}}{\rho_{\mathrm{s}}}\left(\sqrt{\varepsilon_{\mathrm{s}}}-1\right)+1
$$

em que se levou em consideração a densidade do solo $\left(\rho_{\mathrm{b}}\right)$ e a densidade de partículas $\left(\rho_{\mathrm{S}}\right)$ em $\mathrm{Mg} \mathrm{m}^{-3}$, além de considerar as componentes da constante dielétrica aparente do solo $\left(\varepsilon_{\mathrm{S}}\right)$ e da água $\left(\varepsilon_{\mathrm{w}}\right)$ em $\Omega$.

O modelo potencial testado foi obtido com o uso de diversos pares de dados da constante dielétrica aparente e de umidade, que foram submetidos ao aplicativo Table Curve 2D, resultando no modelo potencial do tipo:

$$
\theta=-63,621343+105,03097 \varepsilon^{0,1734662}
$$

O desempenho dos modelos de ajuste dos dados de umidade e constante dielétrica aparente do solo foi avaliado pela análise de variância dos coeficientes de determinação, tendo como variável resposta os dados dos coeficientes de determinação; com isto, definiu-se o modelo mais adequado para estimar a constante dielétrica aparente em função da umidade.

O efeito do tipo de guia de onda (com e sem capacitor) e do número de hastes na determinação da constante dielétrica aparente do solo, obtida pela leitura da TDR, foi avaliado pela análise de variância e pela comparação entre a umidade medida e estimada a partir da constante dielétrica aparente obtida pelo modelo de melhor desempenho. A avaliação do efeito do espaçamento entre hastes foi feita pela análise de regressão, com base nas médias dos desvios-médios entre os valores de umidade medida e estimada.

\section{RESULTADOS E DISCUSSÃO}

A Tabela 1 apresenta os valores das impedâncias médias de três repetições ou de três guias de onda de mesmo padrão de construção para duas e três hastes sob os quatro espaçamentos propostos, considerando-se as guias de onda com e sem capacitor. Os valores das impedâncias foram, em geral, maiores para as guias de onda de duas hastes, o que, de certa forma, era esperado, uma vez que não foram usados dispositivos de ajuste nem casadores de impedância nessas guias de

\begin{tabular}{|c|c|c|c|c|c|c|c|c|}
\hline \multirow{4}{*}{$\begin{array}{l}\text { Tipo de Guia } \\
\text { de Onda }\end{array}$} & \multicolumn{8}{|c|}{ Espaçamentos (m) } \\
\hline & \multirow{2}{*}{\multicolumn{2}{|c|}{$\frac{0,009}{\mathrm{~N}^{\mathrm{o}} \text { hastes }}$}} & \multirow{2}{*}{\multicolumn{2}{|c|}{$\frac{0,013}{\mathrm{~N}^{\mathrm{o}} \text { hastes }}$}} & \multirow{2}{*}{\multicolumn{2}{|c|}{$\frac{0,017}{\mathrm{~N}^{\mathrm{o}} \text { hastes }}$}} & \multirow{2}{*}{\multicolumn{2}{|c|}{$\frac{0,022}{\mathrm{~N}^{\mathrm{o}} \text { hastes }}$}} \\
\hline & & & & & & & & \\
\hline & 2 & 3 & 2 & 3 & 2 & 3 & 2 & 3 \\
\hline $\begin{array}{c}\text { Sem capacitor } \\
\mathrm{Z}_{0}(\Omega)\end{array}$ & 234 & 178 & 292 & 222 & 319 & 231 & 339 & 247 \\
\hline $\begin{array}{c}\text { Com capacitor } \\
\mathrm{Z}_{0}(\Omega)\end{array}$ & 257 & 226 & 294 & 200 & 325 & 226 & 354 & 242 \\
\hline
\end{tabular}
onda, o que as torna susceptíveis a variações de voltagem e corrente.

Tabela 1. Valores médios da impedância característica das guias de onda avaliadas

Para o mesmo número de hastes, a impedância tendeu a aumentar com o espaçamento entre as hastes, cujo aumento se deve à redução do confinamento da onda, o que reduz a similaridade das condições da guia de onda com o cabo coaxial. A variação da impedância, para um mesmo tipo de guia de onda, foi maior no caso sem capacitor com duas hastes, com uma diferença entre a maior impedância (espaçamento entre hastes de 0,022 m) e a menor (espaçamento entre hastes de $0,009 \mathrm{~m}$ ) de $45 \%$.

Essa diferença para o mesmo tipo de guia de três hastes foi de $39 \%$. No caso das guias de onda com capacitor, a diferença entre a maior e a menor impedância foi de $38 \%$ para duas hastes e de $7 \%$ para três hastes. As guias de onda de três hastes são mais adequadas à determinação de umidade, uma vez que apresentaram menores impedâncias, isto é, mais próximas de $200 \Omega$. Guias de onda com valores superiores a $200 \Omega$, tendem a apresentar uma reflexão que prejudica a interpretação do sinal eletromagnético, problema que deve agravar em solos secos e salinos (Souza et al, 2001). Este comportamento, entretanto, não foi observado nas guias de onda avaliadas, mesmo com impedâncias acima de $300 \Omega$. A análise de variância dos dados dos coeficientes de determinação gerados pelos modelos de calibração ou de ajuste de um modelo aos dados da constante dielétrica aparente em função da umidade, mostrou que houve efeito significativo $(\mathrm{p}<0,05)$ do tipo de modelo (Tabela 2).

Tabela 2. Teste de Scott-Knott (1974) para as médias estimadas dos coeficientes de determinação obtidos pelos diferentes modelos avaliados*

\begin{tabular}{lc}
\hline \multicolumn{1}{c}{ Modelo } & $\mathrm{R}^{2}$ Médio \\
Malicki et al. (1996) & $0,6811 \mathrm{a}$ \\
Potencial & $0,5505 \mathrm{~b}$ \\
Roth et al. (1990) & $0,5456 \mathrm{~b}$ \\
Topp et al. (1980) & $0,4032 \mathrm{c}$ \\
Whalley et al. (1993) & $0,3512 \mathrm{c}$ \\
\hline Médias seguidas de mesma letra não diferem a 5\% de probabilidade
\end{tabular}


A média dos coeficientes de determinação (Tabela 2) obtida com uso do modelo de Malicki et al. (1996) para a densidade do solo de $1,33 \mathrm{~kg} \mathrm{dm}^{-3}$, densidade de partículas de $2,50 \mathrm{~kg} \mathrm{dm}^{-3} \mathrm{e}$ o valor médio de $\alpha=0,429$, constituiu um grupo diferente dos outros formados pelos modelos Potencial e de Roth et al. (1990) e pelos modelos de Topp et al. (1980) e de Whalley et al. (1993), tendo apresentado o maior valor, o que indicou o melhor desempenho desse método. Este foi o modelo de maior estabilidade, apresentando as menores médias dos desviosmédios entre os valores da constante dielétrica aparente estimados e medidos nas guias de onda durante todo o período de coleta de dados.

O modelo potencial do tipo $\theta=105,03097 \varepsilon^{0,17346621}+$ 63,621343 , obtido pelo aplicativo Table curve 2D, destacou-se entre os demais quanto aos valores dos coeficientes de determinação, cuja média não diferiu estatisticamente do modelo de Roth et al. (1990), mas diferiu e foi superior à dos modelos de Topp et al. (1980) e de Whalley (1993) que, por sua vez, não diferiram entre si. Esses resultados não coincidiram com os de Coelho et al. (2001) que obtiveram o modelo exponencial como de melhor desempenho na estimativa da umidade em função da constante dielétrica aparente em relação ao modelo de Roth et al. (1990) simplificado e ao de Topp et al. (1980).

O modelo de Malicki et al. (1996), apesar de sua natureza empírica, foi superior ao de Roth et al. (1990), de natureza mais física, por envolver todas as fases do solo, isto é, sólida, líquida e gasosa. O diferencial do modelo de Malicki et al. (1996) consiste na densidade do solo. Com respeito ao expoente da constante dielétrica aparente, $\alpha$, foi considerado o valor de 0,429, inferior a 0,5 mais comumente usado (Topp et al., 1980; Ledieu et al.,1986; Herkelrath et al.,1991; Malicki et al.,1996). O modelo de Whalley (1993), apesar de considerar a densidade do solo e envolver as constantes dielétricas da água e do solo separadamente, foi o modelo de pior desempenho, com menor média de coeficiente de determinação. É um modelo mais adequado para solos arenosos (Robson et al., 1999).

A análise de variância dos dados de constante dielétrica aparente não foi significativa $(\mathrm{p}>0,05)$ para tipo de guia de onda e número de hastes avaliadas isoladamente, o que indicou não ter havido diferença significativa entre as médias das constantes dielétricas aparentes determinadas pelas guias de onda com e sem capacitor, e entre as médias das constantes dielétricas aparentes determinadas pelas guias de onda de duas e de três hastes. Este resultado está em concordância com os obtidos por Coelho et al. (2001) em que não se verificou diferença significativa entre guias de onda com e sem capacitor na estimativa da constante dielétrica aparente. Por outro lado, o efeito do número de hastes era esperado, uma vez que o aumento do número de hastes tende a uniformizar a forma do campo magnético entre as hastes, confinando mais a onda (Conciani et al., 1997); além disso, a guia de onda de três hastes apresentou menor impedância característica e tem grandes vantagens sobre as de duas hastes, principalmente quanto à apresentação do gráfico do pulso eletromagnético, de melhor definição do sinal, que permite detectar facilmente o final da haste pela reflexão do pulso (Noborio, 2001).

Ocorreu efeito do espaçamento entre hastes na determinação das constantes dielétricas aparentes $(\mathrm{p}<0,05)$ sendo que os desvios entre os valores de umidade estimados e medidos para as guias de onda com espaçamento de $0,009 \mathrm{~m}$, foram pelo menos $100 \%$ superiores aos obtidos para as guias de onda com espaçamento de $0,022 \mathrm{~m}$. A análise de regressão dos desvios-médios entre os valores de umidade estimados e medidos (Figura 1) para os diferentes espaçamentos entre hastes, mostrou que o espaçamento de $0,017 \mathrm{~m}$ foi o de maior precisão na obtenção da umidade ou constante dielétrica aparente. Esses resultados não concordam com os de Zegelin et al. (1989) cuja variação do espaçamento entre hastes não interferiu na constante dielétrica da água. Todos os espaçamentos condicionaram a razão diâmetro/espaçamento das hastes para valores superiores a 0,1 , o que concorda com a recomendação de Knight (1992). No caso, a razão mais adequada no dimensionamento das hastes foi a de diâmetro/espaçamento $=0,1764$ correspondente ao espaçamento entre hastes de $0,017 \mathrm{~m}$.

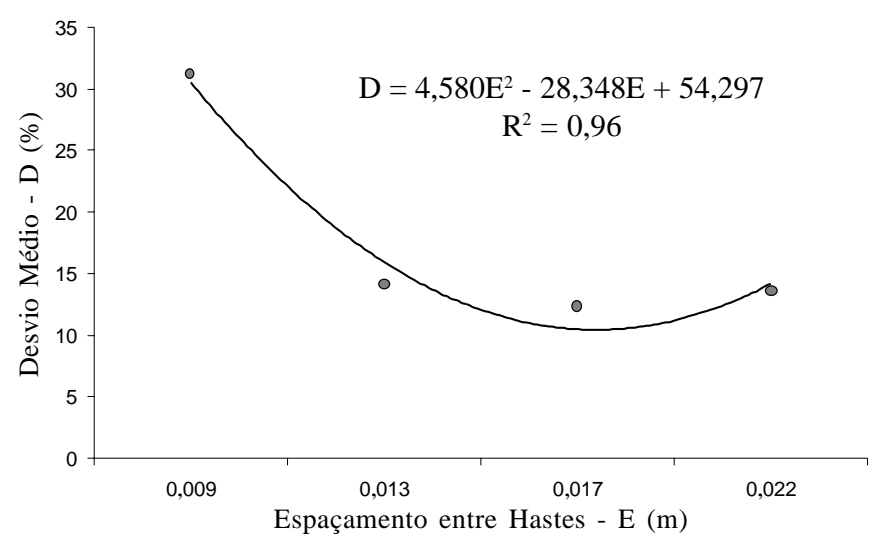

Figura 1. Curva da análise de regressão para os dados das médias dos desvios-médios entre as umidades estimadas e medidas durante a secagem do solo

A análise de variância dos dados das constantes dielétricas aparentes foi significativa $(\mathrm{p}<0,05)$ para a interação tipo de guia de onda e número de hastes. A avaliação das interações mostrou, para guias de onda sem capacitor, que a média das constantes dielétricas aparentes geradas pelas guias de onda de duas hastes $(18,95)$ diferiu significativamente da média das constantes dielétricas aparentes geradas pelas guias de onda de três hastes $(21,47)$ a 5\% de probabilidade pelo teste de ScottKnott (1974). A estimativa do coeficiente da regressão linear para as antenas de três hastes foi mais próximo da unidade, com menor desvio-médio entre os valores de umidade média estimados e medidos (Figura 2) indicando as guias de três hastes como as mais precisas para obtenção da umidade do solo, no caso das guias sem uso do capacitor.

As umidades médias estimadas em função da constante dielétrica aparente pelas guias de onda sem capacitor com três hastes, superestimaram as umidades médias medidas em média $6,4 \pm 5,4 \%$, enquanto as guias de onda com duas hastes subestimaram as umidades médias medidas em média 18,7 \pm $5,2 \%$.

A interação tipo de guia de onda e número de hastes, especificamente o desdobramento tipo de guia e guia de três hastes, foi significativa, sendo que a média das constantes dielétricas aparentes obtidas com guias de onda sem o capacitor 


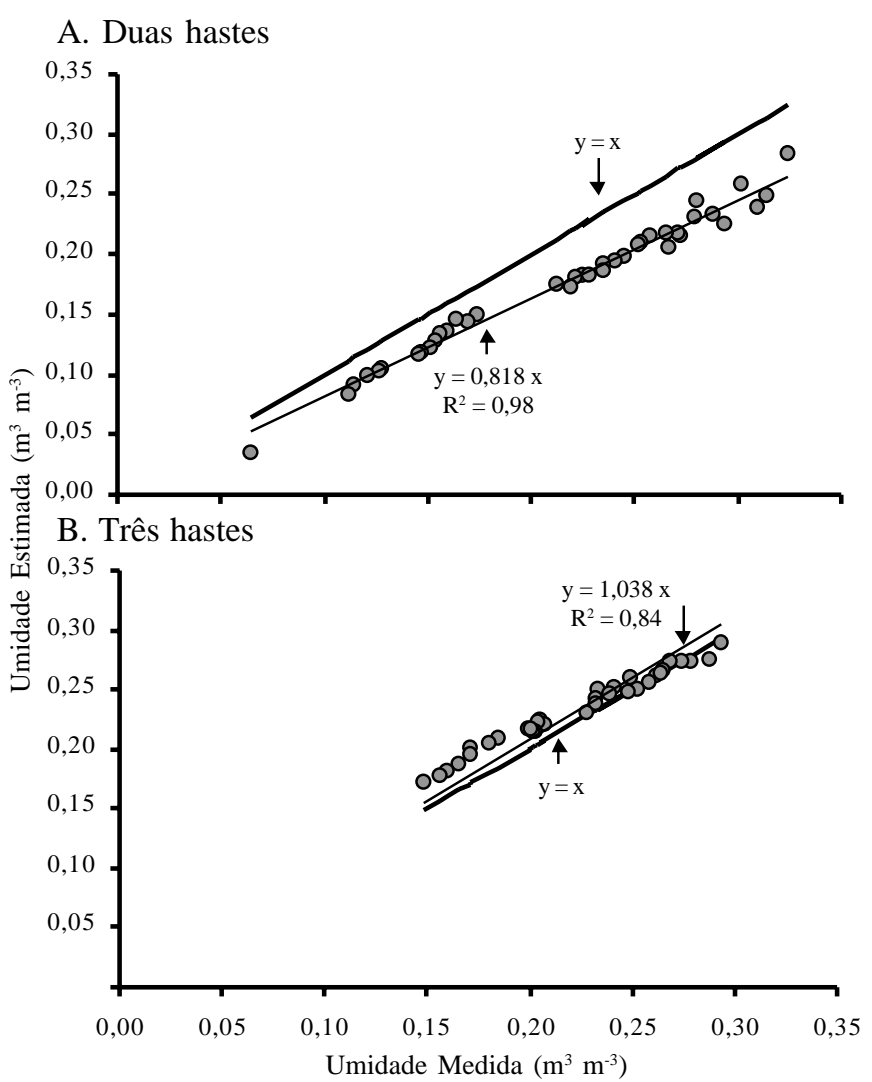

Figura 2. Comportamento da umidade média estimada em função da constante dielétrica aparente, pelas guias de onda de duas (A) e três hastes (B) sem capacitor em relação à umidade média medida por gravimetria

$(21,47)$ diferiu significativamente da média obtida com as guias com o capacitor $(19,42)$ ao nível de $5 \%$ de probabilidade.

As guias de onda sem capacitor estimaram a umidade com maior precisão, com coeficiente de linearidade da regressão umidade, estimada em função da umidade medida mais próximo da unidade $(1,038)$ e com menor desvio-médio entre as umidades medidas e estimadas $(6,4 \% \pm 5,4 \%)$ comparadas às guias com capacitor de três hastes (Figura 3 ) que apresentaram coeficiente de linearidade de 0,888 e desvio-médio entre as umidades medidas e estimadas, de $9,3 \% \pm 4,8 \%$.

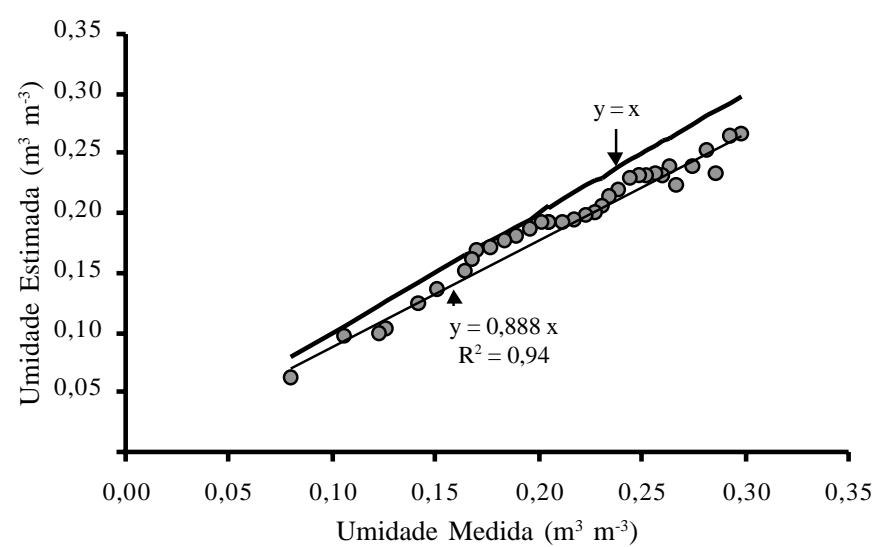

Figura 3. Comportamento da umidade média estimada em função da constante dielétrica aparente pelas guias de onda de três hastes com capacitor, em relação à umidade média medida por gravimetria

\section{CONCLUSÕES}

1. As guias de onda de três hastes de diâmetro $0,003 \mathrm{~m}$, comprimento $0,15 \mathrm{~m}$ e com espaçamentos entre hastes de 0,009 e $0,022 \mathrm{~m}$, tiveram valores de impedância inferiores aos das guias de onda de duas hastes.

2. O modelo de Malicki apresentou o melhor desempenho na determinação da umidade do solo, em função da constante dielétrica aparente.

3. As guias de onda de três hastes espaçadas $0,017 \mathrm{~m}$ entre si foram as de melhor desempenho quanto à estimativa da umidade em função da constante dielétrica aparente.

4. As guias de onda de três hastes sem capacitor apresentaram melhor desempenho na determinação da umidade do solo comparado com as de duas hastes sem capacitor.

5. As guias de onda de três hastes sem capacitor apresentaram melhor desempenho na determinação da umidade do solo que as de três hastes com capacitor.

\section{AGRADECIMENTOS}

Ao Dr. Claudinei F. Souza, pelo apoio na determinação das impedâncias características das guias de onda.

\section{LITERATURA CITADA}

Coelho, E.F.; Andrade, C.L.T.; Or, D.; Lopes, L.C.; Souza, C. F. Desempenho de diferentes guias de ondas para uso com o analisador de umidade Trase. Revista Brasileira de Engenharia Agrícola e Ambiental, Campina Grande, v.5, n.1, p.81-87. 2001.

Conciani, W.; Carneiro, B.J.; Soares, M.M.; Hermann, P.S.P.; Crestana, S. Emprego de TDR com sondas multi-hastes segmentadas para medida de umidade de um perfil de solo. In: Simpósio Nacional de Instrumentação Agropecuária, I, 1997, São Carlos, Anais...São Carlos: EMBRAPA-CNPDIA, 1997. p.169-173.

Herkelrath, W.N.; Hamburg, S.P.; Murphy, F. Automatic, realtime monitoring of soil moisture in a remote field area with time domain reflectometry. Water Resources Research, Washington, v.27, p.857-864, 1991.

Kelly, S.F.; Selker, J.S.; Green, J.L. Using short soil moisture probes with high-bandwidth time domain reflectometry measurements. Soil Science Society of America Journal, Madison, v.59, p.97-102, 1995.

Knight, J.H. Sensitivity of time domain reflectometry measurements to lateral variations in soil water content. Water Research Research. Washington, v.28, p.2345-2352. 1992.

Ledieu, J.; De Ridder, P.; De Clerck, P.; Dautrebande, S., A method measuring soil water moisture by time-domain reflectometry. Journal of Hydrology, Amsterdam, v.88, p.319-328, 1986.

Malicki, M.A.; Plagge, R.; Renger, M.; Walczak, R.T. Application of time-domain reflectometry (TDR) soil moisture miniprobe for the determination of unsaturated soil water characteristics from undisturbed soil cores. Irrigation Science, New York, v.13, p.65-72, 1992. 
Malicki, M.A.; Plagge, R.; Roth, C.H. Improving the calibration of dielectric TDR soil moisture determination taking into account the solid soil. European Journal of Soil Science, Oxford, v.47, p.357-366, 1996.

Malicki, M.A.; Skierucha, W.M. A manually controlled TDR soil moisture meter operating with 300 psi rise-time needle pulse. New York, v.10, p.153-163, 1989.

Noborio, K. Measurement of soil water content and electrical conductivity by time domain reflectometry: a review. Computers and Electronics in Agriculture, Amsterdam, v.31, p. 213-237, 2001

Or, D.; Wraith, J.M. Agricultural and environmental soil physics. Logan: Utah State University.1993. 218p.

Patterson, D.E.; Smith, M.W. The measurement of frozen water content by time domain reflectometry: results from laboratory tests. Canadian Geotechnical Journal, Ottawa, v.18, p.131144, 1981.

Petersen, L.W.; Thomsen, A.; Moldrup, P.; Jacobsen, O.H.; Rolston, D.E. High-resolution time domain reflectometry: sensitivity dependency on probe design. Soil Science, Baltimore, v.159, n.3, p.149-155, 1995.

Robinson D.A.; Gardner, C.M.K.; Cooper, J.D. Measurement of relative permittivity in sandy soils using TDR, capacitance and theta probes: comparison, including the effects of bulk soil electrical conductivity. Journal of Hydrology, Amsterdam, v.223, p.198-211, 1999.
Roth, C.H.; Schulin, R.; Fluhler, H.; Attinger, W. Calibration of time-domain reflectometry for water content measurement using composite dieletric approach. Water Resources Research, Washington, v.26, n.10, p.2267-2273, 1990.

Scott, A.J.; Knott, M.A. A cluster analysis method for grouping means in the analysis of variance. Biometrics, Washington, v.30, n.3, p.507-512, 1974.

Souza, C.F.; Matsura, E.E.; Testezlaf, R. Avaliação de sonda de TDR multi-haste segmentada na determinação do bulbo molhado em irrigação localizada. In: Congresso Brasileiro de Engenharia Agrícola, 29, 2000, Fortaleza: SBEA/UFC, 2000. CD-Rom

Souza, C.F.; Matsura, E.E.; Testezlaf, R. Electromagnetic determination of water content: Measurements in multi-wire probes with electrical impedance discontinuities. In: International Microwave and Optoelectronics Conference, 2001, Belém. IEEE, 2001.

Topp, G.C.; Davis, J.L.; Annan, A. Electromagnetic determination of soil water content: measurement in coaxial transmission lines. Walter Resources Research, Washington, v.16, p.576583, 1980.

Whalley, W.R. Considerations on the use of time domain reflectometry (TDR) for measuring soil water content. Journal of Soil Science, Edinburgh, v.44, n.1, p.1-9. 1993.

Zegelin, S.J.; White, I.; Jenkins, D.R. Improved field probes for soil water content and electrical conductivity measurements using TDR. Water Resources Research, Hanover, v.25, p.2367-2376, 1989 . 